\title{
LA PARTICIPACIÓN DE LA MUJER EN LAS DISTINTAS FORMAS DE RESISTENCIA DURANTE LA OCUPACIÓN DE GRECIA POR LAS FUERZAS DEL EJE (1940-1944)
}

\author{
WOMEN'S PARTICIPATION IN DIFFERENT FORMS \\ OF RESISTANCE DURING THE OCCUPATION OF \\ GREECE BY THE AXIS POWERS (1940-1944)
}

Maila García Amorós

Universidad de Granada

\section{RESUMEN}

Desde los primeros momentos de la Ocupación las mujeres se organizaron para emprender acciones destinadas a paliar la situación que trajo a la nación la invasión de las fuerzas del Eje o para combatir al invasor directamente. En un primer momento su participación en actos de Resistencia, podrían considerarse una extensión de las labores que tradicionalmente había desempeñado, haciéndose cargo de la atención de los soldados y de la población civil que había permanecido en los pueblos y ciudades. Paulatinamente, las mujeres fueron asumiendo roles hasta ese momento restringidos a los hombres. Fueron muchas las que a través de diversas agrupaciones participaron activamente en la Resistencia. Muchas de ellas emprendieron iniciativas particulares mediante las cuales salvaron numerosas vidas o infringieron daño al enemigo. No faltaron, por otro lado, las que incorporándose al ejército lucharon como partisanas y tomaron parte en la batalla al lado de los hombres. Objetivo de este trabajo es destacar la labor de todos estos grupos de mujeres que de un modo u otro presentaron resistencia contra el invasor.

Palabras clave: Ocupación, resistencia, mujer, partisana, emancipación.

\begin{abstract}
From the very beginning of the Occupation, women organized themselves to carry out acts in order to alleviate the situation brought upon the nation by the Axis powers, or to directly fight against the invader. Initially, their participation in these actions could be considered an extension of the tasks they had traditionally carried out but they were no less important because of that. In this way,
\end{abstract}


they began to take care of the soldiers and civilians who stayed in the villages and towns. The women gradually took on roles which until then had been restricted to men. Many women enlisted and contributed to the resistance struggle through the different groups they established. There was also an important number of women who were posted in the mountains and took part in the fighting alongside the men. Nor should we forget those who undertook specific initiatives, by means of which they saved many lives or inflicted injury on the enemy. The aim of this essay is to highlight the work of all these groups of women who in one way or another showed resistance against the invader.

Keywords: Ocupation, resistence, women, partisan, emancipation.

\section{RESUM \\ LA PARTICIPACIÓ DE LA DONA EN LES DIFERENTS FORMES DE RESISTÈNCIA DURANT L'OCUPACIÓ DE GRÈCIA PER LES FORCES DE L'EIX (1940-1944)}

Des dels primers moments de l'Ocupació, les dones s'organitzaren per a emprendre accions destinades a pal.liar la situació que va portar a la nació a la invasió de les forces de l'Eix o per a combatre a l'invasor directament. En un primer moment la seua participació en actes de Resistència, podria considerar-se una extensió de les tasques que tradicionalment havien desenvolupat, fent-se càrrec de l'atenció dels soldats i de la població civil que havia restat en els pobles i ciutats. Gradualment, les dones assumiren rols fins eixe moment restringits als homes. Van ser moltes les que a través de les diverses agrupacions participaren activament a la Resistència. Moltes d'elles van emprendre iniciatives particulars mitjançant les quals salvaren nombroses vides o van infringir mal a l'enemic. No faltaren, per altra banda, les que incorporant-se a l'exèrcit lluitaren com a partisanes i van prendre part a la batalla al costat dels homes. L'objectiu d'aquest treball és destacar la feina de tots aquests grups de dones que d'una manera o altra presentaren resistència contra l'invasor.

Paraules clau: Ocupació, resistència, dona, partisana, emancipació. 
"Cuando hablamos de Resistencia, nuestra mente no ha de ir sólo a la lucha armada, al frente y a las montañas. Cuando hablamos de Resistencia nacional no nos referimos sólo a aquellos que participaron activamente en el combate o que pertenecieron a organizaciones. Entre los miembros de la Resistencia no hemos de contar sólo a aquellos que se jugaron la vida todos los días o a los osados jóvenes que iban a la vanguardia. Cuando hablamos de Resistencia griega, no nos referimos sólo a los prisioneros, a los rehenes, a los ejecutados, sino que incluimos a todos aquellos que se opusieron de alguna manera al invasor. Incluimos a aquellos que con su desobediencia a las órdenes del invasor, con su negativa y su oposición a aceptar cualquiera de sus disposiciones, no se rendían sino que luchaban. Resistencia presentó el combatiente, pero también aquel que no creyó en lo que dictaba el opresor. Presentó resistencia aquel que daba un trozo de pan al hambriento y un vaso de agua al sediento para calmar su sed".

1 GLEZOS, Manolis (2009), EӨvıkń Avtíotaбıs, tomo I, Stochastís, Atenas, p. 61. 
Como señala con estas palabras el autor de uno de los primeros actos de resistencia en la Grecia ocupada, Manolis Glezos, ${ }^{2}$ la Resistencia no consistió sólo en la lucha armada o en las acciones de sabotaje llevadas a cabo contra el invasor, sino que también hemos de considerar actos de resistencia todos aquellos gestos y acciones gracias a los cuales esta lucha pudo sostenerse. Nos referimos con esto a las muchas iniciativas de las personas que permanecieron en las ciudades y los pueblos para hacer frente a la situación, paliar los efectos de la invasión y combatir, cada uno en la medida de sus posibilidades, al invasor. La mayoría de estas iniciativas fueron promovidas por mujeres, que fueron quienes se quedaron al cargo, no sólo de sus propias familias, sino también de la población civil en general. A lo largo de estas páginas, iremos viendo en qué consistió esta labor y cómo paulatinamente las mujeres fueron involucrándose en actividades hasta entonces llevadas a cabo por hombres, como la colaboración en la Resistencia y la participación como partisanas en las guerrillas.

\section{LA INVASIÓN DE GRECIA Y SU ENTRADA EN LA SEGUNDA GUERRA MUNDIAL}

Tras el estallido de la guerra en Europa, el dictador griego loannis Metaxás, ${ }^{3}$ en el poder desde 1936, trató de llevar a cabo una política neutral tanto con Inglaterra como con Alemania, con la voluntad de mantener a Grecia apartada del conflicto. Sin embargo, las provocaciones por parte de Italia para que Grecia entrara en la guerra eran continuas. A principios de 1940 Italia torpedeó el Crucero griego Elli, mientras que el 28 de octubre de 1940 el gobierno de Mussolini lanzaba un ultimátum al gobierno griego, según el cual éste debía ceder a los italianos algunas bases militares y territorios de los que harían uso hasta el final de la guerra. Este ultimátum fue rotundamente rechazado por Metaxás, quien no pudo evitar

2 La noche del 30 de mayo de 1941, dos jóvenes estudiantes escalaron la roca en la que se erige la Acrópolis de Atenas, arriaron la bandera alemana con la esvástica que los alemanes habían colocado tras su entrada en Atenas e izaron la bandera griega. Este fue considerado el primer acto de resistencia contra el invasor. Uno de esos jóvenes era Manolis Glezos, quien continuó participando en actos de resistencia durante toda la Ocupación y fue detenido y torturado en varias ocasiones, logrando siempre salvar su vida. Después de la guerra civil griega (1946-1949) se dedicó a la política militando en la izquierda y siendo diputado en varias ocasiones, la última en 2014. A día de hoy, a sus 96 años, Siguió participando en mitines políiticos y publicando libros y artículos de opinión en la prensa griega hasta su muerte a los 97 años, el 30 de marzo de 2020, durante el confinamiento del COVID-19.

3 Esta negativa rotunda de Metaxás, conocida como "El Gran No", sigue celebrándose hoy día en Grecia como fiesta nacional cada 28 de octubre. 
que pocas horas más tarde, las tropas italianas pasaran por la fuerza a Grecia a través de la frontera con Albania y el país entrara así en la guerra del lado de los aliados. ${ }^{4}$

Inmediatamente después de la declaración de guerra, el ejército griego se concentró en la frontera de Albania, donde logró frenar a las tropas italianas y las hizo retroceder. Los éxitos militares del ejército griego fueron tales, que llegaron a conquistarse algunas ciudades fronterizas de Albania en las que había una importante población griega. Sin embargo, la llegada del invierno puso fin a las campañas militares. Entretanto, Inglaterra había ofrecido colaboración a Grecia, colaboración que fue rechazada por Metaxás en la idea de que, de esta manera, podría evitar el conflicto con Alemania. ${ }^{5}$ No obstante, al morir Metaxás en enero de 1941, su sucesor, Aléxandros Korizis, no tuvo tales miramientos. La ayuda prometida por los ingleses llegó, pero muy tarde, cuando ya no había forma de frenar a las tropas alemanas que finalmente entraron a Grecia por la frontera con Yugoslavia y Bulgaria el 6 de abril de 1941.6

En medio del caos que sobrevino, Aléxandros Korizis se suicidó. La mayor parte de la fuerza expedicionaria inglesa fue evacuada junto con el Gobierno y la Casa Real, que marcharon a Creta con el fin de proteger la isla de la invasión y organizar desde allí la Resistencia. Sin embargo, la isla cayó poco tiempo después, en mayo del 41 , tras una sangrienta batalla de la que las fuerzas alemanas salieron debilitadas. ${ }^{7}$ La Casa Real y el Gobierno marcharon entonces a Oriente Próximo, estableciéndose en Grecia un gobierno colaboracionista encabezado por el general Yorgos Tsolázoglu. ${ }^{8}$ A principios de junio de 1941, la totalidad de Grecia se encontraba ya sometida por Alemania, que ocupó Atenas, Macedonia, la frontera con Turquía y parte de la isla de Creta, por Italia, que ocupó el Peloponeso y

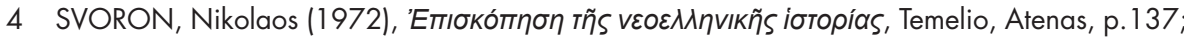

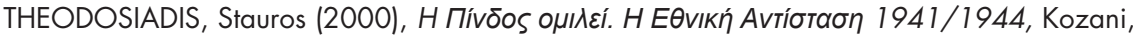

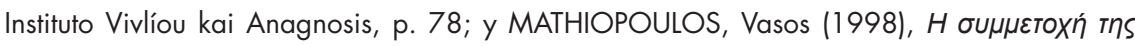

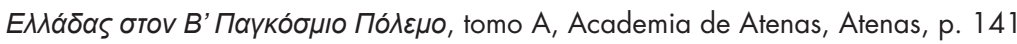

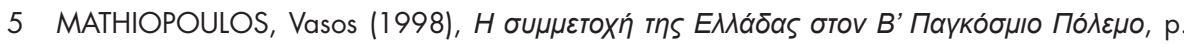
256.

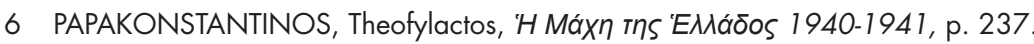

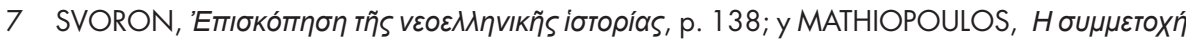

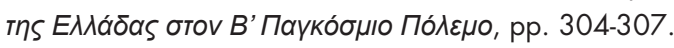

8 El 23 de abril, tres días antes de la entrada de las tropas en Atenas, Yorgos Tsolázoglu había pactado con los alemanes un armisticio sin la autorización gubernamental. Tras la marcha de la familia real y el contingente británico, formó un gobierno colaboracionista. CLOGG, Richard (1998), Historia de Grecia, Cambridge University Press, Cambridge, p. 121. 
Grecia Central y por Bulgaria, a quien se le permitió ocupar la Tracia Occidental y parte de Macedonia, donde la población griega fue víctima de persecuciones y exterminios. ${ }^{9}$

\section{LABOR ASISTENCIAL DE LAS MUJeres tRAS LA ENTRADA DE GRECIA EN LA GUERRA}

Poco después del primer acto simbólico de resistencia perpetrado por Manolis Glezos y Apóstolos Santas con el izado de la bandera griega en la Acrópolis, comenzó a gestarse la idea de una Resistencia griega. En julio de 1941 habían tenido lugar algunas reuniones clandestinas con el fin de analizar la situación y empezar a organizarse. Sin embargo, los desacuerdos surgidos entre los líderes de las distintas orientaciones políticas dieron al traste con la idea de una Resistencia sólida y unida. ${ }^{10}$ Aprovechando esta situación de desacuerdo, el Partido Comunista (KKE) creó la agrupación EAM, ${ }^{11}$ de la que a finales de 1941 surgiría el ELAS, ${ }^{12}$ su brazo militar, que comenzó a desarrollar una intensa actividad. Otros oficiales del ejército griego fueron fundando grupos de resistencia no comunistas como el EDES, ${ }^{13}$ bajo el mando del general Napoleón Zervás, quien se apostó

9 La cifra de víctimas griegas a manos de los búlgaros ascendía a unas 15.000 , tras las matanzas colectivas perpetradas en Drama, Kavala, Doxato y otros pueblos. VAKALÓPULOS, Apóstolos (1995), Historia de Grecia Moderna (1204-1985), Universidad de Chile, Santiago, p. 324; y CONDOGEORGIS, Georgios (1992), Histoire de la Grèce, Hatier, París, p. 405.

10 VAKALOPULOS, Historia de Grecia moderna, p. 325; y GASPARINATOS, Spyros (1998), 'H

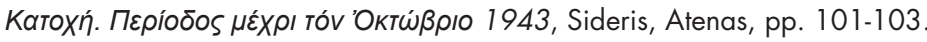

11 El EAM (Frente Nacional de Liberación) tenía dos objetivos fundamentales, en primer lugar, organizar la Resistencia y, en segundo lugar, consolidar una posición política preponderante que le permitiera, una vez terminada la guerra, asumir el poder político. CLOGG, Historia de Grecia, pp. 122 y 125; VAKALÓPULOS, Historia de Grecia moderna, p. 326; SVORON, iñs

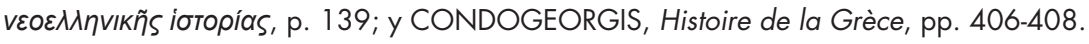

12 El ELAS (Ejército Popular de Liberación Nacional) aprovechó el vacío político originado en tiempos de Metaxás y su experiencia en la clandestinidad para organizarse como la mayor fuerza militar de la Ocupación. Esta ambición militar, pero también política, llevó al ELAS a numerosas confrontaciones y ataques contra otros grupos de la Resistencia no comunistas. CLO-

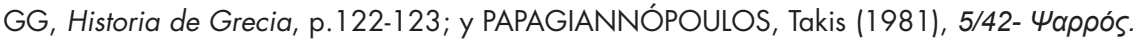

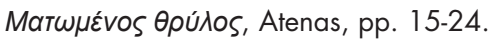

13 El EDES (Ejército Nacional Democrático Griego) fue la segunda agrupación de la Resistencia más fuerte después del EAM. Era de inspiración venizelista y socialista y se posicionó en contra de la monarquía, aunque posteriormente su líder Napoleón Zervás se declararía a favor del regreso del rey Jorge II. Entre sus logros más importantes destaca la destrucción del viaducto de Gorgopótamos el 25 de noviembre de 1942, que puso freno al paso de los suministros del Mariscal Rommel por Grecia. CLOGG, Historia de Grecia, pp. 219-220; y GASPARINATOS, 'H Kaтохи́, pp. 307-310. 
en las montañas a mediados 1942 y la EKKA, ${ }^{14}$ liderada por el coronel Dimitrios Psarós.

Con la marcha de los hombres al frente enrolados en las distintas agrupaciones de la Resistencia, las mujeres no tardaron en organizarse para tratar de cubrir las numerosas carencias de organización y medios materiales. Ya hacia 1939, en previsión de la guerra que se avecinaba, se habían puesto en marcha escuelas de enfermería, donde muchas jóvenes acudían a formarse a fin de prestar sus servicios en la guerra. Entre los organismos que ofrecían esta formación cabe destacar el Instituto de la Mujer Griega, ${ }^{15}$ que se comprometió desde el principio con la causa del apoyo al ejército. De esta manera, en octubre de 1940, 2.836 mujeres con formación en enfermería siguieron al ejército griego al frente de Albania para asistir a los soldados heridos o enfermos. Estas enfermeras, movilizadas por la Cruz Roja Griega, dotaron los 58 hospitales militares, las unidades avanzadas de salud pública, los hospitales móviles y los centros de primeros auxilios. ${ }^{16}$ El apoyo material a los soldados y guerrilleros constituyó desde el principio una de las preocupaciones más inmediatas tras el estallido de la Guerra. Iniciativa del Instituto de la Mujer Griega fue también la institución Franela para el Soldado, cuyo fin era proveer de ropas y mantas al ejército griego apostado en las montañas, donde el frío del invierno estaba haciendo estragos. Esta iniciativa se puso en marcha por un grupo de mujeres presidido por Elmina Votsi ${ }^{17}$ y funcionaba bajo los auspicios de la entonces princesa Federica. Franela para el soldado constituyó el principal esfuerzo

14 EKKA (Liberación Nacional y Social) fue una agrupación de inspiración socialdemócrata fundada por el coronel venizelista Dimitrios Psarrós. Llevó a cabo grandes avances militares, logrando liberar la zona de Ánfisa en noviembre del 43. Sin embargo, repetidos enfrentamientos con el ELAS llevaron a su disolución y al asesinato de su líder y fundador. PAPAGIANNÓ-

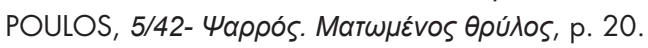

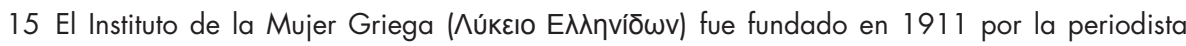
Calirroe Parén, una de las primeras feministas griegas, con el objetivo de combatir la xenofilia y preservar los usos y costumbres de Grecia. Ya en el periodo de entreguerras era un vestigio del antiguo feminismo y considerado bastante conservador. AVDELA, Efi y PSARRÁ, Angélica



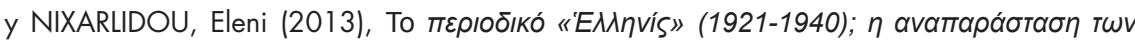

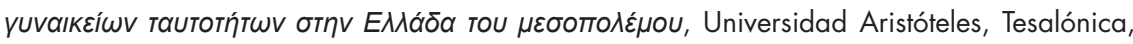
Tesis doctoral, p. 31.

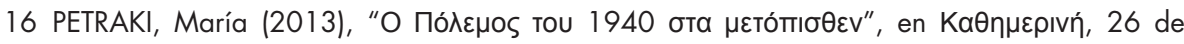
octubre de 2013 [https://www.kathimerini.gr/502233/article/epikairothta/ellada/o-polemos-toy-1940-sta-metopis8en] (última consulta 8 de julio de 2019).

17 La institución fue fundada ya en 1938 por Elimna Votsi, Alexandra M. Melá y Amalia Likou- 
y también el más efectivo para suplir las enormes carencias indumentarias del ejército griego ${ }^{18}$ e involucró a la inmensa mayoría de la población griega femenina de todos los rincones del país. ${ }^{19}$ Desde señoras de las grandes ciudades hasta aldeanas de las zonas agrícolas más aisladas, todas participaron aportando lo que cada una podía: materias primas, como lana de sus propias ovejas, sus telares para la elaboración de los tejidos o sus manos para la confección de las prendas. Se ha calculado que la producción de ropas y mantas ascendía a las tres mil piezas diarias. ${ }^{20}$

En lo que se refiere al apoyo material, no podemos dejar de hacer alusión a las mujeres de Pindos, cordillera montañosa al sureste de la frontera albanesa, que cargaron con el armamento y las provisiones para los soldados apostados allí y se lo hicieron llegar por caminos intransitables por donde no era posible el paso de las mulas que solían transportar estos materiales. ${ }^{21}$ En las ciudades, las mujeres asumieron la administración, la protección y el sustento de la población. Su actividad en un principio fue de naturaleza puramente asistencial y consistió en una extensión de los roles tradicionales que habían venido desempeñando y que pasaron del ámbito particular del hogar y la familia al ámbito general y más extenso de los pueblos y ciudades y de la población civil. Esta labor, sin embargo, no debe subestimarse, pues era completamente necesaria y fue determinante para la protección y la supervivencia de muchos colectivos en situación de vulnerabilidad, como los niños y los ancianos.

rezou, y en un principio de dedicó al dar aliento a los soldados mediante cartas y envíos de pequeños paquetes con comida u objetos de utilidad en el frente. No obstante, hacia 1939, cuando el estallido de la guerra parecía ya inminente, empezó a desarrollar una importante actividad destinada a dotar al ejército de una indumentaria adecuada al clima y a la oro-

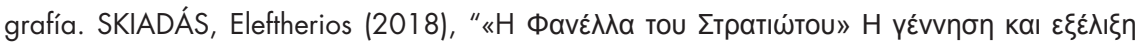

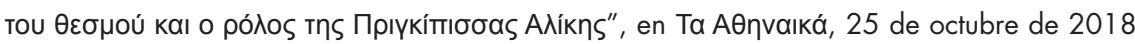
[https://www.taathinaika.gr/i-fanella-tou-stratiotou/].

18 Los uniformes del ejército griego eran de la Primera Guerra Mundial con polainas y botas que no se adecuaban al terreno montañoso donde se combatía. Había además una gran escasez

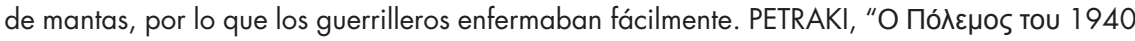
бта $\mu \varepsilon т о ́ т ı \sigma \theta \varepsilon v^{\prime \prime}$.

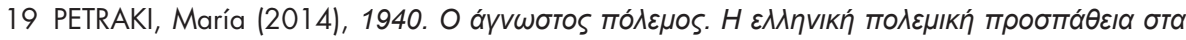

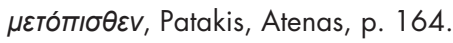

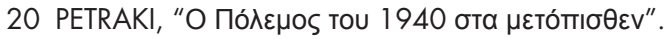

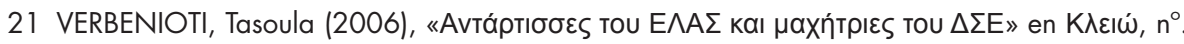
3, Epikentro, Tesalónica, p. 163. 
Lo más urgente e inmediato era alimentar a la población, sobre todo durante el periodo de la gran hambruna del invierno de 1941 y $1942 .{ }^{22}$ Una de las primeras iniciativas llevadas a cabo en Atenas para intentar paliar esta situación fue la institución Vida al niño, ${ }^{23}$ que consistió en organizar un sistema de atención a los niños, de modo que cada familia pudiente del barrio de Plaka se encargara de dar al menos una comida diaria a un niño. Este sistema fue concebido por un grupo de mujeres del ateniense barrio de Plaka, encabezado por loanna Tsatsos ${ }^{24}$ y funcionó durante todo el periodo de hambruna en Atenas. La propia loanna Tsatsos, se encargó poco después, y con el amparo del Arzobispado de Atenas, de poner en marcha el primer comedor público. ${ }^{25}$ Poco a poco y también por iniciativas individuales o colectivas de mujeres, se fueron abriendo comedores públicos en otras ciudades y pueblos de toda Grecia. El abastecimiento de estos comedores se hacía por medio de colectas o de intercambios. En Euritania, por ejemplo, las jóvenes que se ocupaban del comedor preparaban representaciones teatrales, para asistir a las cuales pedían al público que

22 Tras la entrada de los alemanes, las reservas de alimentos y las materias primas de Grecia, así como sus principales fuentes de ingresos habían sido requisadas como botín de guerra, de manera que la población griega quedó rápidamente desabastecida. Los productos de primera necesidad que se producían en Grecia eran trasladados a Alemania o acababan en el mercado negro. Esta situación acabó causando, pocos meses después de la entrada de los alemanes, la total devaluación de la moneda e imponiendo el sistema del trueque. La consecuencia más grave de todo ello fue, sin duda, la gran hambruna que se desató y que se cobró unas 300.000 víctimas en el invierno de 1941 y 1942. VAKALÓPULOS, Historia de Grecia, p. 322; GASPARINATOS, 'H Katoxń, pp. 46-56; y CONDOGEORGIS, Histoire de la Grèce, p. 406.

23 Esta institución estuvo presidida por loanna Tsatsos y constituida por Rita Liambei como secretaria, Irini Tsimbuki, Alexandra Miloná, Irini Jrisikopoulou, Lena Zaná, Miranda Ikonomou, Pan-

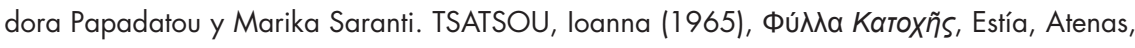
p. 32.

24 loanna Tsatsos era hermana del poeta Georgios Seferis. Nació en Esmirna en 1902 y se trasladó a Atenas con su familia en 1914. Tras la ocupación de Grecia por las fuerzas del eje, llevó a cabo actividades de apoyo a la Resistencia como facilitar la huida de oficiales aliados o trasladar información entre sus miembros. Junto con su marido Constantinos Tsatsos, colaboró estrechamente con la agrupación EKKA, así como con el coronel loannis Tsigantes. Llevó a cabo también una importante labor social y humanitaria en colaboración con el arzobispo de Atenas Damaskinós. En 1965 publicó su obra Diario de la Ocupación, un interesante documento sobre ese periodo de la historia de Grecia. GARCÍA AMORÓS, Maila, Autobiografía e historia en la obra de loanna Tsatsos, Universidad de Granada, Granada, 2008.

25 TSATSOU, Фú $\lambda \lambda \alpha$ Katoxñ , р. 30. 
llevara alubias o maíz para abastecer los comedores en lugar de pagar una entrada. ${ }^{26}$

No podemos dejar de hablar en este punto de la importante labor humanitaria llevada a cabo en el seno del Arzobispado de Atenas, donde se desarrolló una intensa actividad de apoyo a las familias. Tras los primeros fusilamientos, que empezaron en Grecia el 5 de octubre de 1941, pronto se hizo evidente la situación de indefensión y de necesidad en la que quedaban sus familias. ${ }^{27}$ De esta manera, el arzobispo Damaskinós concibió la idea del Servicio de Asistencia a las Familias de los Ejecutados, que empezó a funcionar en marzo de 1942. Damaskinós ${ }^{28}$ delegó la dirección del Servicio en loanna Tsatsos, quien a su vez se rodeó de un grupo de mujeres que lo gestionó durante toda la Ocupación. ${ }^{29}$ Este servicio consistía en llevar un minucioso registro de las personas que ingresaban en prisión y de aquellas que eran ejecutadas, con la finalidad de poder facilitar la información necesaria a las familias. ${ }^{30}$ El objetivo último, no obstante, era establecer un sistema de pensiones para las familias de los rehenes y de los ejecutados. De esta manera, en un principio, el Arzobispado dio cober-

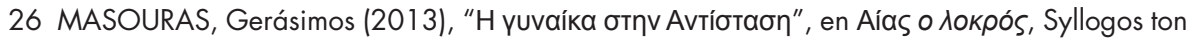
en Athinais kai Apantaxou Martinaion, n' 33 [http://www.martino.gr/images/pdf/AIAS_33. pdf) (última consulta 07 de julio de 2019).

27 La situación de necesidad en que quedaban las familias y la tremenda hambruna de 1941 empujaron a muchas mujeres a la prostitución. Como sucedió también en otros países ocupados, aquellas que ofrecieron sus servicios a soldados $u$ oficiales alemanes o italianos pagaron tras la Ocupación un precio muy caro, la exclusión social y el escarnio público, entre otros

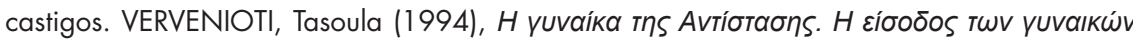

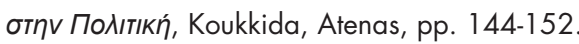

28 Dimitrios Papandreou nació en Ełolia en 1891. Se ordenó sacerdote en 1917, adoptando el nombre de Damaskinós. Fue nombrado arzobispo de Atenas tras la entrada en Grecia de las fuerzas del Eje. Al frente de este cargo llevó a cabo una importante actividad en favor de las víctimas de la Ocupación y de los judíos, por lo que fue declarado uno de los «Justos entre las Naciones». Al finalizar la Ocupación ejerció el cargo de Primer Ministro en funciones.

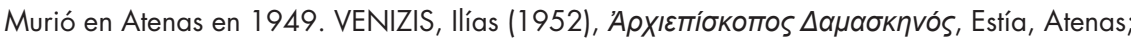

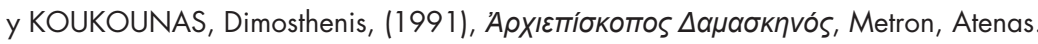

29 Entre estas mujeres se encontraban Lilika Teotokás, Anna Alevrás, Sosó Stupatis, Lula Trilivas, María Kútsikos y María Papadakis. TSATSOU, Фú $\lambda$ a Katoxñs, p. 85.

30 La lista de ejecutados elaborada por loanna Tsatsos sirvió como prueba en los Juicios de Nuremberg y se publicó en 1947 con el título de Ejecutados durante la Ocupación. TSATSOU,

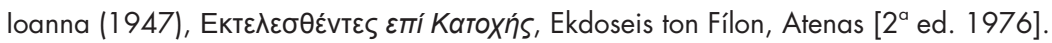


tura económica a numerosas familias atenienses. Algo más tarde, a partir de 1943, estas pensiones empezaron a concederse de manera legal ${ }^{31}$ y pudieron llegar a muchas familias de las provincias. El dinero se obtenía a través de colectas organizadas por el grupo de mujeres a cargo del servicio. ${ }^{32}$ La concesión de estas ayudas molestaba tremendamente a los mandos italianos, quienes sospechaban que el arzobispo estaba recibiendo dinero de Oriente Medio. En 1943, el propio Damaskinós fue sometido a arresto domiciliario y loanna Tsatsos fue llamada a un interrogatorio, del que pudo salir indemne. ${ }^{33}$

Dentro de la labor humanitaria llevada a cabo por el Arzobispado, no podemos dejar de aludir a sus esfuerzos por salvar a la población judía. Cuando el 17 de abril de 1943 comenzó la persecución de judíos en Grecia, con la noticia de que todo hebreo que no fuera de origen griego debía marcharse a su país y que los hebreos griegos debían marchar a Polonia, Damaskinós, junto con 27 representantes políticos y académicos, firmó una carta en la que se hablaba de «los griegos de la comunidad judía» y defendía que éstos debían ser considerados griegos sin distinción alguna de raza o de religión. loanna Tsatsos, que estuvo en esos momentos al lado del arzobispo, da testimonio de los certificados de nacionalidad griega falsos y de las partidas bautismales que entregaban a los judíos a fin de hacerlos pasar por griegos ortodoxos. ${ }^{34}$

En relación con las iniciativas de socorro a las víctimas de la Ocupación, hemos de destacar la labor desempeñada por la organización Solidaridad Nacional, que estuvo funcionando desde el principio de la Ocupación y en la que participaron mujeres de todos los lugares del país. Solidaridad Nacional surgió por inspiración del EAM y se fundó en mayo de 1941. En sus comienzos se dedicó a ofrecer apoyo y facilitar la huida a los griegos perseguidos, a organizar visitas a las prisiones para llevar ropas y alimentos a los presos y a paliar los efectos de la hambruna de 1941-1942. Paulatinamente, la agrupación fue ampliando su campo de actuación y empezó a prestar también servicios médicos y educativos. De esta manera, se fundaron hospitales para la atención de los soldados heridos, pero también de la población civil, así como guarderías y escuelas para garantizar la educación durante este periodo, principalmente en las zonas rurales. ${ }^{35}$

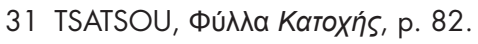

32 TSATSOU, Фú $\lambda$ a Katoxńs, p. 72.

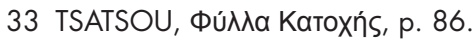

34 De los 62.700 hebreos que vivían en Grecia antes de la Ocupación, sobrevivieron tan sólo 10.000 .

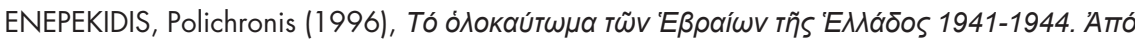

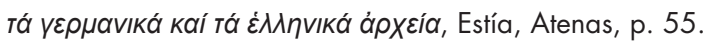

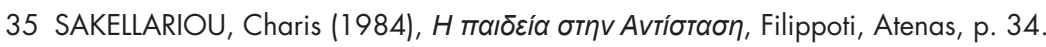


La Solidaridad Nacional se extendió rápidamente por toda la geografía griega, desde las ciudades más importantes, hasta los pueblos más remotos y estaba integrada en su inmensa mayoría por mujeres. La participación en la Solidaridad Nacional estaba relativamente bien considerada por la sociedad, porque se veía como el modo más adecuado en que una mujer podía contribuir a la lucha, ya que podían hacerlo sin salirse de sus roles tradicionales. La organización alcanzó su mayor número de participantes el último año de la Ocupación, hecho que se ha asociado precisamente a la revalorización del papel de la mujer. ${ }^{36}$ Hay que añadir, no obstante, que para colaborar con esta organización, las mujeres tuvieron que superar numerosos prejuicios, no sólo por parte de los hombres y de la sociedad en general, sino también por parte de las propias mujeres de otras generaciones que no comprendían su participación en la lucha.

\section{Participación de las mujeres en la resistencia}

Conforme se prolongaba la Ocupación, las mujeres fueron involucrándose de manera cada vez más activa en la lucha por la liberación y participando en actividades tradicionalmente restringidas a los hombres. Fueron muchas las que, abandonando sus hogares y rompiendo, en muchas ocasiones, los lazos familiares, ${ }^{37}$ se enrolaron en diversas agrupaciones de la Resistencia. Como ya señalamos al comienzo, el EAM era, con diferencia, la agrupación más fuerte de la Resistencia. Hacia finales de 1941 se había creado su brazo militar, el ELAS, que en diciembre de 1942 se había extendido ya por gran parte de Grecia y en cuyas filas fueron enrolándose rápidamente no sólo partisanos de ideología comunista, sino también muchos otros de otras ideologías. El EAM, a pesar de su actitud hostil hacia otras agrupaciones de la Resistencia, ${ }^{38}$ contó con un enorme apoyo popular que se granjeó gracias a sus promesas de un futuro más halagüeño para las gentes de las áreas rurales, para los jóvenes y para las mujeres. ${ }^{39}$ Del EAM surgieron dos

36 VERVENIOTI, H yuvaíka tns Avtíotaons, p. 229.

37 No fueron pocas las mujeres de familias acomodadas y conservadoras que se enrolaron en agrupaciones de Resistencia de izquierdas, lo que provocaba, en la mayoría de los casos, una enorme brecha en sus relaciones familiares.

38 Representativos de la actitud hostil del ELAS con los otros grupos de Resistencia son los numerosos ataques perpetrados contra ellas, como el de octubre de 1943 contra el EDES o los continuos ataques contra la EKKA, provocando su desintegración en varias ocasiones y que culminarían con el asesinato de su líder, Dimitrios Psarós el 17 de abril de 1944. Estos ataques ponían en evidencia su fuerte deseo de hacerse con el poder político una vez terminada la guerra. CLOGG, Historia de Grecia, p. 220; PAPAGIANNÓPOULOS, 5/42$\theta$ oúlos, p. 84; y CONDOGEORGIS, Histoire de la Grèce, p. 408.

39 CLOGG, Historia de Grecia, p. 128. 
agrupaciones de juventudes que en sus comienzos disgregaron a hombres de mujeres. Así, la agrupación Joven Libre estaba constituida exclusivamente por mujeres, mientras que el EAM Joven estaba formado únicamente por varones. Ambas agrupaciones terminarían confluyendo en febrero de 1943 en una sola que se llamó EPON. ${ }^{40}$

Joven Libre, cuyo nombre revelaba claramente tanto las promesas del EAM con respecto a la emancipación como las expectativas de las jóvenes que se incorporaban a la agrupación, se gestó en el ámbito universitario y brindaba a sus jóvenes integrantes la oportunidad de cambiar su modo de vida y de asumir nuevas responsabilidades más allá de aquellas a las que parecían estar destinadas. No hay que olvidar que hablamos, en la mayoría de los casos, de mujeres que provenían de familias conservadoras -las familias que podían costear estudios a sus hijas eran también las más conservadoras de la sociedad-sujetas a tradiciones ancestrales que mantenían a la mujer atada al matrimonio y al cuidado del hogar y de los hijos. La agrupación fue creciendo en número de integrantes y extendiéndose por las provincias incluyendo en sus filas a mujeres de toda Grecia, que deseaban contribuir a la lucha y reivindicar, de este modo, que también ellas eran capaces de organizarse y de emprender acciones por la liberación de la nación. La agrupación estuvo dirigida por un importante miembro de la Resistencia, Electra Apostolou (1912-1944), responsable también de la elaboración del material propagandístico ilegal del Partido Comunista, lo que le costó ser apresada y ejecutada por miembros de la Seguridad Especial Griega $^{41}$ en julio de 1944. La participación de la mujer en actos de resistencia asociados a esta agrupación fue activa y consistía fundamentalmente en hacer pintadas en las calles o repartir propaganda ilegal, transportar armas y alimentos o transmitir información y mensajes. Algunas, como es el caso de Hero Constantopoulou participaron también en actos de sabotaje. Además de la agrupación Joven Libre, en Atenas operaba también la orga-

40 En el EPON (Organización Panhelénica Unida de Juventudes) terminarían por confluir también numerosas organizaciones de Resistencia nacionales y regionales que decidieron la desintegración de todas ellas para formar una sola organización.

41 La Dirección de Seguridad Especial del Estado, más conocida como Seguridad Especial, era un ala de las Fuerzas de Seguridad griegas creado en 1929 con objeto de perseguir las organizaciones comunistas. Durante la Ocupación colaboró estrechamente con las fuerzas de Ocupación y participó en detenciones, torturas y ejecuciones de miembros de la Resistencia griega. Fue desintegrada en septiembre de 1944. GEORGÍOU, Vasos (ed.) (1979), Iбторía ins Avtíotaons 1940-45, Aulós, Atenas, p. 1442. 
nización de sabotaje PEAN, ${ }^{42}$ que registró una alta participación femenina. Digno de mención es el caso particular de loulía Bimba (†1943), una sencilla empleada de hogar ${ }^{43}$ procedente de Samos, quien tras ingresar en el PEAN, ofreció su casa como taller para la fabricación de bombas. Ella misma fue la encargada de transportar hasta el centro de Atenas y de colocar la bomba de 10 kilos con la que se voló la sede de la ESPO ${ }^{44}$ en diciembre de 1942. Este hecho es considerado uno de los más importantes actos de sabotaje infringidos al enemigo. loulía Bimba fue apresada junto con otros miembros de la organización y ejecutada a principios de 1943. En relación con la participación de la mujer en la Resistencia, no se puede dejar de hablar de Eleni Karagianni (1898-1944), más conocida con el hipocorístico de Lela, considerada una de las grandes figuras de la Resistencia griega. Lela Karagianni creó y lideró una de las primeras agrupaciones de Resistencia que empezaron a operar en Grecia y la llamó Bubulina. ${ }^{45}$ Se trataba de una pequeña, pero sumamente eficaz organización, integrada por unas 150 personas, cuyo núcleo lo conformaban ella misma, su marido Nikólaos Karagiannis y sus siete hijos. La organización se dedicó principalmente a facilitar la huida de oficiales británicos atrapados en la

42 El PEAN (Unión Panhelénica de Jóvenes Cambatientes) fue una pequeña organización de la Resistencia constituida por pocos miembros y dedicada al sabotaje. Estaba dirigida por Constantinos Perikos y era de inspiración progresista y socialista. Entre sus golpes más importantes destaca la explosión de la sede del ESPO. LIMPERATOS, Michalis (2007), Oı opyavẃozıs thS Avtíotaons, t.III, parte II, Vivliorama, Atenas, p. 50; y HATZIVASILÍOU, Evanthis (2004), ПEAN

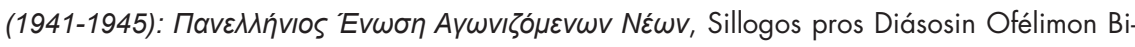
blion, Atenas, p. 114.

43 Aunque loulía Bimba es conocida como "la maestra de la Resistencia", en realidad no era maestra. No obstante, durante la Ocupación impartía clases en su tiempo libre en la escuela de San Nicolás, de ahí que se la conozca con este apelativo. VATAKIS, Pantelís, LAGÓS,

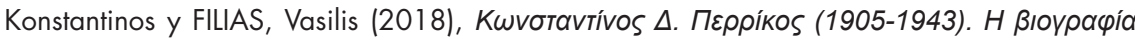
Evós ńpwa, Menandros, Atenas, p. 654.

44 La ESPO (Organización Nacional Socialista y Patriota) había sido creada por los alemanes con la finalidad de enrolar a griegos y crear un pelotón colaboracionista que luchara contra Rusia del lado de los alemanes. GASPARINATOS, 'H Катохи́, p. 238

45 El nombre elegido era el de la célebre heroína de la Guerra de la Independencia griega Laskarina Bubulina (1771-1825) que, al mando de su propio buque, el Agamenón, sembró el terror entre la flota otomana en el Mar Sarónico. No en vano, además, Lela Karagianni era bisnieta de Laskarina Bubulina. Para más información sobre Lela Karagianni y la agrupación Bubulina

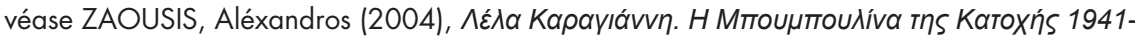
1944, Okeanida, Atenas. 
Grecia Ocupada. ${ }^{46}$ Es preciso señalar que la ayuda, que los miembros de la Resistencia ofrecían a los aliados, los ponía en serio peligro, sobre todo a partir del 8 de diciembre de 1941, cuando los alemanes comenzaron a aplicar una nueva ley, según la cual, quien fuera descubierto escondiendo o prestando auxilio a los ingleses sería ejecutado. ${ }^{47}$ Lela Karagianni fue, de hecho, detenida acusada de estar escondiendo a oficiales aliados y pasó siete meses en prisión antes de ser liberada por falta de pruebas. Al poco de ser liberada retomó su actividad al mando de la agrupación. Con recursos propios, se hizo con cuatro barcos en los que trasladaba clandestinamente, no sólo a oficiales aliados, sino también a griegos perseguidos y a judíos cuando empezaron las persecuciones en Grecia. La agrupación Bubulina tendió, además, una importante red de espionaje. Lela Karagianni poseía una personalidad increíblemente carismática y logró granjearse la colaboración y el apoyo de algunos oficiales alemanes e italianos contrarios al nazismo. El objetivo de esta red de espionaje era, entre otros, recabar información sobre los movimientos de los buques alemanes, sustraer planos de aeropuertos y transmitir a sus colaboradores información sobre los mandos de la Ocupación. La agrupación tenía también contacto con agentes del ejército aliado en Oriente Medio. A partir de 1943, empezó a prestar su colaboración a la organización de sabotaje APOLON. ${ }^{48}$ En julio de 1944 fue traicionada y detenida, para ser ejecutada poco después, tan sólo un mes antes de la retirada de las tropas alemanas de Grecia. Lela Kariagianni coincidió en prisión con la jovencísima Hero Constantopoulou (1927-1944) de 17 años, miembro de la agrupación EPON, que había sido detenida por su participación en la explosión de un tren que transportaba armamento alemán. Aunque pertenecían a agrupaciones de distinta ideología, entre ambas surgió una fuerte relación de amistad. Hero Constantopoulou fue ejecutada tres días más tarde que Karagianni.

La participación de las mujeres en la Resistencia no se limitó a la capital griega, sino que también en las provincias se emprendieron iniciativas, ya en el seno de las agrupaciones existentes, ya a nivel particular, como fue el caso de María Dimadi que merece la pena mencionar. María Dimadi (1907. 1944) procedía de una familia acomodada de Agrinio, en Etolo-Acarnania,

46 Aunque el contingente británico había sido evacuado con el gobierno en abril de 1941, meses más tarde todavía quedaban muchos oficiales ingleses dispersos que no habían podido huir antes de la entrada de las tropas alemanas.

47 VAKALÓPULOS, Historia de Grecia, p. 325.

48 La organización de sabotaje APOLON se dedicó a volar buques enemigos hacia finales de

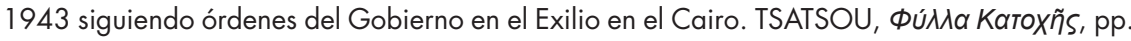
$119-121,166,167$ y 173. 
y había recibido una formación esmerada, por lo que dominaba varias lenguas, entre ellas, el alemán. Cuando los alemanes entraron en Agrinio y pidieron un intérprete, María Dimadi acudió voluntaria, lo que le dio acceso a mucha información sobre la estrategia militar alemana en la zona. María sacaba la información del cuartel alemán en las hojas de carboncillo que utilizaba para hacer las copias de los documentos que mecanografiaba. A través del pope de la ciudad, Constantinos Papavalis, que hacía de enlace, estos documentos oficiales llegaban hasta los guerrilleros y miembros de la Resistencia de la zona de Agrinio. Fue de esta manera como la Resistencia (el ELAS), informada del ataque sorpresa que los alemanes preparaban en la zona, pudo organizarse, atajar el ataque e infringir un daño importante al enemigo el 10 de julio de 1943. Esta fue, de hecho, la primera empresa exitosa de la Resistencia griega en la zona de Agrinio. Tras estos hechos, María pasó a estar en el punto de mira, no sólo de los alemanes, sino también de los Batallones de Seguridad, ${ }^{49}$ quienes finalmente la apresaron y la ejecutaron. ${ }^{50}$

\section{LAS PARTISANAS}

En la lucha por la liberación no faltaron tampoco mujeres que, tras recibir instrucción militar, se incorporaron como oficiales a las filas de guerrilleros y combatieron junto a ellos tomando parte en los ataques de las guerrillas. El ELAS había creado una Escuela de Oficiales en la que en 1941 había matriculados 350 hombres y 2 mujeres. Sin embargo, hacia el final de la guerra, el número de mujeres matriculadas había ascendido a 65 . Entre las filas del ELAS se encontraban 13 mujeres que se habían licenciado en la Escuela de Oficiales de Rentina con el grado de subteniente y que se hacían cargo de la gestión de los pelotones de mujeres. ${ }^{51}$ Hay que señalar, en este punto, que pasar a formar parte de las filas de partisanos del ELAS

49 Los batallones de seguridad se crearon en abril de 1943 y eran unidades militares integradas por voluntarios griegos y armadas por Alemania con el fin de combatir a los grupos de guerrilleros del EAM. Los primeros voluntarios eran gentes sin recursos que trataban así de evitar ser enviados a Alemania, criminales o miembros amenazados por la Resistencia. Empezaron a crecer, sobre todo, tras la rendición italiana en septiembre de 1943. Los Batallones de Seguridad colaboraron en el traslado de judíos griegos a Auschwitz y en general sembraban el terror entre la población civil, ya que junto a la Seguridad Especial tomaban rehenes que eran ejecutados como represalia de los ataques de la Resistencia. MAZOWER, Mark (1995), Inside Hitler's Greece: The Experience of Occupation (1941-44), Yale University Press, New Haven, pp. 334-358.

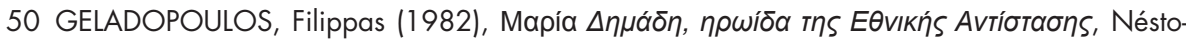
ras, Atenas.

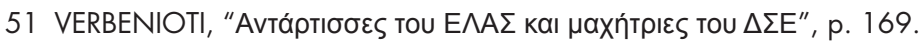


no resultaba fácil para las mujeres, ya que además de la autorización de los mandos del propio ejército del ELAS, necesitaban una autorización paterna. Evidentemente muchos padres se negaban a mandar a sus hijas a la guerra. Para ser consideradas partisanas necesitaban, además, disponer de su propia arma, cosa que tampoco resultaba fácil, pues el ejército no proporcionaba armas a mujeres. La mayoría de ellas tuvieron que procurarse su propia arma. ${ }^{52}$ Es paradigmático el caso de Melpómene Papailíou, apodada Thyela (tempestad), que portaba una automática y unos pantalones que le había quitado a un soldado alemán al que había dado muerte. En un primer momento, las guerrilleras participaron de manera desorganizada junto con los hombres. Posteriormente se fueron organizando en pelotones constituidos exclusivamente por mujeres. Estas mujeres guerrilleras se convirtieron así en una especie de propaganda para la exhortación a la lucha, pues constituía una forma de demostrar que podían actuar $y$, por lo tanto, que las gentes de los pueblos (hombres y mujeres) podían sumarse también a la lucha contra el invasor. Este tipo de propaganda servía además para tratar de lavar el nombre del EAM, tras sus numerosos ataques contra agrupaciones de Resistencia no comunistas.

La actividad de las guerrilleras que integraban los pelotones estaba muy unida a la de los jóvenes guerrilleros del EPON. Sus obligaciones eran militares, políticas y culturales. Evidentemente, tomaban parte en las batallas junto con los hombres, se hacían cargo de la vigilancia de la División, hacían rondas en los pueblos e informaban a las gentes sobre el avance de la guerra. Las guerrilleras colaboraban también organizando representaciones culturales, teatros, bailes, etc. y a la vez recogían provisiones. Una vez creado el EPON en 1943, no hubo disgregación de género y hombres y mujeres convivían como iguales, lo cual pronto creó un fuerte espíritu de camaradería entre ellos. Existía, no obstante, una ley no escrita, según la cual no podían establecer relaciones amorosas entre ellos hasta que no terminara la guerra..$^{53}$ En el material fotográfico conservado de aquella época puede verse a las jóvenes partisanas con el cabello recogido a fin de no constituir una provocación para sus compañeros varones. Esta norma se mantuvo con mucha disciplina y severidad, tanto es así, que en la División 13 expulsaron a una muchacha por considerar que su belleza constituía una provocación para los oficiales del ejército local. Así mismo, en septiembre de 1944 en una zona recién liberada, una guerrillera fue acusada de mantener relaciones sentimentales con un hombre y condenada a deponer 
el arma. La condena suponía un agravio tan grande, que la muchacha se suicidó con su propia arma antes de que se la quitaran. ${ }^{54}$

Aunque la participación de las mujeres en las guerrillas se ha asociado normalmente a convicciones de izquierdas, ${ }^{55}$ es preciso señalar que no todas estas mujeres -ni tampoco todos los hombres- abrazaban ideales comunistas, sino que una gran parte de sus integrantes se enrolaban por considerar que era una forma de luchar contra el invasor de manera organizada, independientemente de sus ideas políticas. ${ }^{56}$ Así pues, las mujeres veían en el EAM una forma de abandonar los roles tradicionales a los que estaban sometidas y de emanciparse en una sociedad que, sobre todo en las zonas más rulares, era todavía acusadamente patriarcal. ${ }^{57} \mathrm{~A}$ pesar de ello, muchos de ellos, hombres y mujeres, sufrieron persecuciones y castigos al terminar la guerra y sobre todo tras la derrota comunista en la guerra civil griega que sucedió a la Ocupación. ${ }^{58}$

\section{Conclusiones}

Es muy importante hacer énfasis en que la participación de la mujer en la Resistencia les brindó la oportunidad de asumir nuevos roles y de sentirse no sólo partícipes en la lucha por la liberación de la nación, sino también de su propia liberación. De esta manera, las mujeres emprendieron iniciativas, empezaron a tomar decisiones propias, a reafirmar su autoestima y a desarrollar una nueva identidad femenina, que adquirió nuevas dimensiones: la de combatiente, la de mujer que asume la administración y la gestión de las ciudades y de los servicios, la de quien tiene en su mano salvar la vida de otras personas. Fue de este modo como se hizo patente que las mujeres desempeñaban un importante papel en la vida de un estado. A través de su participación en la Resistencia las mujeres adoptaron, además, conciencia política, de una u otra ideología, y poco a poco fueron asumiendo responsabilidades. Su participación en las decisiones políticas se fue normalizando, no en vano durante la Ocupación las mujeres participaban de las decisiones y votaban igual que los hombres. La primera vez que las mujeres ejercieron su derecho el voto en Grecia fue en las votaciones celebradas en abril de 1944 para la creación de un Consejo Nacional en las zonas de Grecia controladas por el EAM. ${ }^{59}$ Todo ello acabaría abrien-

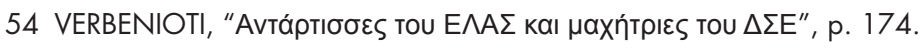

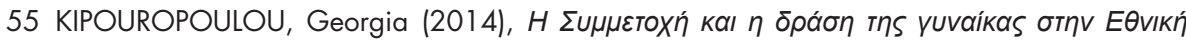

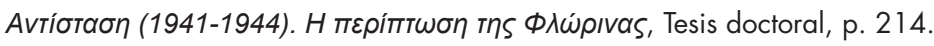

56 VAKALÓPULOS, Historia de Grecia, p. 326.

57 CLOGG, Historia de Grecia, p. 123. 
do finalmente el camino a su emancipación social y al reconocimiento de su derecho al voto en 1956.60

La mujer, no obstante, tendría que superar otro duro obstáculo antes de poder emanciparse y ejercer sus derechos en plena libertad: la guerra civil griega (1946-1949), que dividió al país durante más de tres años y que trajo unas consecuencias funestas. ${ }^{61}$ No cabe ahora detenerse en el papel que desempeñó la mujer en esta otra guerra, la mayoría de ellas, no obstante, siguieron desarrollando actividades parecidas a las que habían estado desarrollando durante la Ocupación, en el marco ahora, de una confrontación civil, con todas las implicaciones que este hecho conlleva. ${ }^{62}$ Podemos afirmar, a modo de conclusión que, con la participación de la mujer en las diversas formas de Resistencia, se superaron numerosas dudas y prejuicios que imperaban en la sociedad hasta ese momento y que las mujeres lograron ganarse en parte el respeto de sus compañeros masculinos abriendo así el camino hacia su emancipación social y su participación política. Durante este difícil momento de la historia de Grecia, habían demostrado con creces que eran capaces de hacerse cargo de la administración de las ciudades, de la protección de las familias, que eran capaces también no sólo de colaborar en actos sabotaje, sino también de crear y dirigir organizaciones de Resistencia y redes de espionaje y finalmente, que eran capaces de luchar al lado de los hombres empuñando su propia arma. En esos momentos, ya nadie podía dudar de la igualdad entre hombres y mujeres, era una igualdad de facto.

58 Precisamente la política vengativa del Partido Popular, vencedor en las primeras elecciones libres tras la Ocupación, acrecentó la crispación política y culminó con la formación de un ejército comunista llamado Ejército Democrático, liderado por Markos Vafiadis, antiguo líder del ELAS, y el estallido de la Guerra Civil Griega, que se prolongó hasta 1949, con la derrota del Ejército Democrático, gracias a la colaboración de los Estados Unidos. MAZOWER, Mark (2000), After the war was over, Princeton University Press, Princeton; y RIZAS, Sotiris (2011),

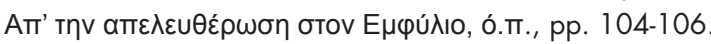

59 CLOGG, Historia de Grecia, p. 128.

60 VERVENIOTI, H yuvaíka ths Avtíotaons, pp. 260-270.

61 Además de las pérdidas humanas, la Ocupación y la posterior guerra civil dejaron al país en una situación lamentable. La marina mercante quedó totalmente destrozada, así como la producción agrícola e industrial y gran parte de las infraestructuras. También fueron arrasados pueblos enteros, de manera que al final de la guerra había cerca de 1.200 .000 personas $\sin$ hogar. CONDOGEORGIS, Histoire de la Grece, pp. 414-415.

62 Muchas de las mujeres guerrilleras pasaron entonces a las filas del Ejército Democrático Grie-

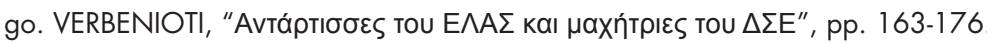

